\title{
Clinical Significance of Perineal Descent in Pelvic Outlet Obstruction Diagnosed by using Defecography
}

\author{
Hyun Nam Baek, Yong Hee Hwang, Yong Hwan Jung \\ Department of Surgery, Sahm Yook Medical Center, Seoul, Korea
}

Purpose: The aim of this study was to evaluate the clinical significance of perineal descent (PD) in pelvic outlet obstruction patients diagnosed by using defecography.

Methods: One hundred thirty-six patients with pelvic outlet obstruction (POO; median age 49 years) had more than one biofeedback session after defecography. Demographic finding, clinical bowel symptoms and anorectal physiological studies were compared for $\mathrm{PD}$ at rest and $\mathrm{PD}$ with dynamic changes.

Results: Age $(r=0.33$; $P<0.001)$, rectocele diameter $(r=0.31 ; P<0.01)$, symptoms of incontinence $(P<0.05)$ and number of vaginal deliveries $(\mathrm{r}=0.46 ; \mathrm{P}<0.001)$ were correlated with increased fixed $\mathrm{PD}$. However, the female gender $(\mathrm{P}<$ 0.005), rectal intussusceptions $(\mathrm{P}<0.05)$, negative non-relaxing puborectalis syndrome $(\mathrm{P}<0.00005)$ and rectocele $(\mathrm{P}<$ $0.0005)$ were correlated with increased dynamic PD. Duration of symptoms, number of bowel movements, history of pelvic surgery and difficult defecation were not related with PD. There was no significant correlation between fixed and dynamic PD and success of biofeedback therapy.

Conclusion: Age, vaginal delivery and diameter of the rectocele are associated with increased fixed PD. Female gender, rectal intussusceptions and a rectocele are correlated with increased dynamic PD. Biofeedback is an effective option for POO regardless of severity of PD.

Keywords: Perineal descent; Pelvic outlet obstruction; Biofeedback

\section{INTRODUCTION}

Perineal descent is a phenomenon associated with constipation, and it is considered to be the last stage of excessive straining for defecation over several years. Such abnormally increased perineal descent was observed by Porter [1] in 1962 for the first time, and afterward, it was accepted as a definite entity by Parks et al. [2] in 1966, and it was described as a relaxed pelvic floor in chronic constipation patients. Subsequently, other investigators [3-5] have reported that increased perineal descent is a general symptom observed not only in constipation patients

Received: August 31, 2010 Accepted: October 20, 2010

Correspondence to: Yong Hee Hwang, Ph.D.

Department of Sugery, Sahm Yook Medical Center, 29-1 Hwigyeong 2-dong,

Dongdaemun-gu, Seoul 130-711, Korea

Tel: +82-2-2210-3563, Fax: +82-2-2249-0403

E-mail: hwangyon@hotmail.com

(C) 2010 The Korean Society of Coloproctology

This is an open-access article distributed under the terms of the Creative Commons Attribution NonCommercial License (http://creativecommons.org/licenses/by-nc/3.0) which permits unrestricted non-

commercial use, distribution, and reproduction in any medium, provided the original work is properly cited. but also in fecal incontinence, idiopathic anal pain syndrome, and solitary rectal ulcer syndrome patients. It is generally accepted to be a secondary phenomenon associated with various diseases causing pelvic outlet obstruction rather than an independent disease inducing chronic constipation.

Pelvic outlet obstruction, together with colonic inertia caused by dysfunction of colon movement, is a major cause, excluding organic causes, of idiopathic chronic constipation. In the past, the representative disease causing pelvic outlet obstruction was anismus; it is a concept referring to entire dyschezia and has been mixed with terms such as pelvic floor dyssynergia, spastic pelvic floor syndrome, paradoxical puborectalis contraction, rectoanal dyssynergia, and nonrelaxing puborectalis syndrome [6]. Nevertheless, on account of the introduction of the anorectal function test, which has been performed widely from the mid-1980s, studies on the functional causes inducing pelvic outlet obstruction have been conducted actively. Based on the results of such studies, presently, non-relaxing puborectalis syndrome, a rectocele, rectal intussusception, sigmoidocele, anal dyschezia are understood to be diseases associated with pelvic outlet obstruction. Therefore we examined the associa- 
tion of the level of perineal descent with pelvic outlet obstruction and the effect of perineal descent on its treatment.

\section{METHODS}

Among patients who visited the Anorectal Clinic at the Sahm Yook Medical Center for symptoms of dyschezia from June 2005 July 2008, the study was conducted on 136 patients who had been diagnosed as having pelvic outlet obstruction by using the anorectal physiological function test and who had received biofeedback treatments for more than 2 times. Pelvic outlet obstruction was diagnosed by using both defecography and cinedefecography. Anal manometry and anal electromyography (EMG) were also performed simultaneously on the patients.

For defecography without pretreatments, patients were put in the left lateral position and the inner wall of the rectum and anal canal were coated by injecting $50 \mathrm{~mL}$ of barium sulfate solution (Solotop, soln; Taejoon Pharm., Seoul, Korea) through the anus, and semi-solid dye prepared in advance was injected into the rectum until the patient felt the sensation of defecation. Afterward, the patient sat on a defecography chair, and under fluoroscopy, radiographs were taken at rest, squeeze, and push. For cinedefecography, the entire defecation process was videotaped with a video camera attached to the fluoroscopy. As a semi-solid dye, barium sulfate suspension (Solotop 120\%) mixed with starch at the ratio of 1:1, was used. Psychological dyschezia factors were minimized by performing all procedures by remote control. The patient was isolated in a test room, and the area was dimmed.

In defecography and cinedefecography, non-relaxing puborectalis syndrome was diagnosed as cases in which clear impressions in the puborectalis muscle are shown during defecation, the antorectal angle is not changed in comparison with the angle at rest or rather becomes smaller, and when defecation is attempted, perineal descent hardly occurs, and the opening of the anal canal and the excretion of more than $80 \%$ feces are delayed for more than 20 seconds. A rectocele whose diameter is bigger than $2 \mathrm{~cm}$ and in which, despite several attempts at defecation, the contrast is not excreted from the rectocele is considered to be a significant rectocele.

For the diagnosis of a sigmoidocele, a first-degree sigmoidocele was defined as one located below the sacral promontory but above the pubococcygeal line. A second-degree sigmoidocele was defined as one located between the pubococcygeal line and the ischiococcygeal line. A third-degree sigmoidocele was defined as one located below the ischiococcygeal line. Sigmoidoceles of higher than second degree were considered to be clinically significant. Rectal intussusception was defined as the rectum showing a funnel-shaped depression within the anal canal during push.

Fixed descent and dynamic descent were measured by using scout film imaging during the rest period and the push period of defecography. A line was drawn on the tangential line of the distal rectal posterior margin and the central axis of the anal canal. The distance to reach the pubococcygeal line, at a right angle, from the point where the above two lines met was measured as the perineal descent. The distance of perineal descent during the rest period was defined as fixed descent, and the difference between the perineal descent during the rest period and that during the push period was defined as dynamic descent.

The device used to measure anal pressure was a water-filled perfusion device. The test catheter was a custom-made polyvinyl catheter $4.5 \mathrm{~mm}$ in diameter that had six side holes; 4 side holes, $0.8 \mathrm{~mm}$ in diameter, were arranged on the same level at $90^{\circ}$ angles, and the other 2 side holes were located within a balloon at the tip of the catheter. The rate of water perfusion was regulated at $0.5 \mathrm{~mL} / \mathrm{channel} / \mathrm{minute}$ by using a microperfusion device with a 15-PSI pressure. The measured pressure was transmitted to the PC Polygraph HR (Sandhill Scientific Inc., Littleton, CO, USA) by using a pressure converter device, re-entered into a computer, and analyzed by using installed software.

For the test, without pretreatment, patients were positioned in the left lateral position. After the pressure of the system had been adjusted, and while taking care to prevent bending, the catheter was placed at a position such that its side hole were located at a site $6 \mathrm{~cm}$ away from the anal verge. Anal manometry was performed using the station pull-through technique, and at $1-\mathrm{cm}$ intervals, including anal manometry at rest, anal manometry was performed at squeeze and at push. The length of the high-pressure zone of the anal canal, in which the pressure was elevated by more than $20 \mathrm{mmHg}$ in more than $50 \%$ of the measured channel (for example, for 4 channels, more than 2 channels) the presence or absence of anorectal inhibitory reflex, the sensory threshold of the lower rectum, and the volume and compliance of the rectum were measured.

For anal electromyography (EMG), without pretreatment, patients were positioned in the left lateral position, a surface electrode $12 \mathrm{~mm}$ in diameter and $45 \mathrm{~mm}$ in length (Perry meter anal EMG sensor EPS-21; Perry Meter Systems, Strafford, PA, USA) was inserted into the anus, and the electric activity levels at rest, squeeze, and push were measured. For biofeedback treatments, the Perry meter anal EMG sensor EPS-21, 12 $\mathrm{mm}$ in diameter and $45 \mathrm{~mm}$ in depth, and a surface electrode were connected to the Kontinence biofeedback computer (HMT Co., Seoul, Korea), and EMG-based biofeedback treatments were performed. The anal EMG sensor was inserted into the anal canal in order to measure the movement of the puborectalis muscle and the anal sphincter. The surface electrode was attached to the abdominal wall in order to measure constriction and relaxation of abdominal muscles as a marker of defecation. The patients were educated concerning the constriction and relaxation of the anal sphincter, and for smooth defecation, 
relaxation of the anal sphincter was learned. In addition, highfiber diets and a sufficient intake of water (more than 8 cups per day) were recommended. The routine use of other laxatives, stimulatory constipation drugs, or suppositories was not allowed. Biofeedback treatments, 30-60 minutes per treatment at one week intervals, were administered by one specialist for all patients who visited outpatient clinics.

After treatments, the patients were asked to evaluate the results by using one of the following classifications: loss of symptoms, improvement of symptoms, continuous symptoms, and worse symptoms. Loss and improvement of symptoms were considered as the treatment success group. Continuous symptoms and worse symptoms were considered as the failure group.

To examine the correlation of perineal descent to pelvic outlet obstruction, we measured, compared and analyzed demographic factors, clinical symptoms including defecation symptoms, the past history of anorectal surgery and obstetrical surgery, and the anorectal physiological function for fixed perineal descent and dynamic perineal descent. In defecography, the anorectal angle at rest, squeeze, and push, the difference between the push period and the rest period, the presence or absence of a rectocele, the size of the rectocele, and the presence or absence of rectal intussusception, sigmoidocele, and non-relaxing puborectalis syndrome were compared and analyzed. In anal electromyography, the electric activities of the puborectalis muscle and the anal sphincter at the rest, squeeze, and push phase were analyzed. To examine the effect of perineal descent on the treatment of pelvic outlet obstruction patients, during fixed perineal descent and dynamic perineal descent, the perineal descent level was determined and analyzed for the success group and the failure group.

For statistical analysis, descriptive statistics, t-test and regression test, were applied. All statistical analyses were performed by using an analysis tool pack (Microsoft Excel 97, 4.00.950,

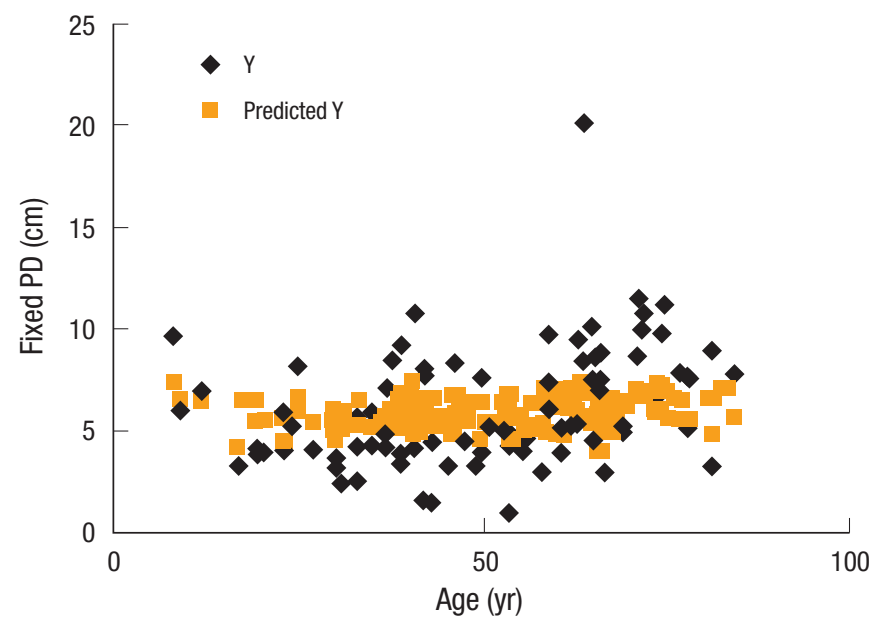

Fig. 1. Age and fixed perineal descent $(P D ; r=0.33, P<0.001)$.
Microsoft Korea, Seoul, Korea). P-values less than 0.05 were considered to be statistically significant.

\section{RESULTS}

The number of patients that visited the Anorectal Clinic at the Sahm Yook Medical Center for dyschezia, were diagnosed as having pelvic outlet obstruction by using defecography, and received biofeedback treatment for more than 1 time was 136 (male:female, 27:109). Their mean age was 49 years (range, 7 to 84 years), and the symptomatic period was an average 6.4 years. The major symptoms of the subjects were dyschezia, sensation of incomplete defecation, hard stool, anal hemorrhage, discharge of mucus, and anal pain. Additional symptoms were decrease in the diameter of stool, an anal lump, abdominal pain, abdominal distention, and a change in defecation habit. Some patients underwent adjuvant defecation therapy, such as laxatives, enemas, and digitations. As for the past disease history, diabetes patients were 6 cases, hypertension patients were 14 cases, back pain patients were 11 cases, patients with disorder of the central nervous system were 3 cases, patients under psychotherapeutic medication were 5 cases, patients under analgesic medication were 7 cases, patients with abdominal surgery unrelated to the rectum were 10 cases, patients with obstetrical surgery were 6 cases, and patients with anorectal surgery were 9 cases. When the disease groups were classified based on the result of the anorectal physiological function test, patients with rectoceles, rectal intussusceptions, sigmoidoceles, and non-relaxing puborectalis syndrome were 77 cases (56\%), 28 cases (20\%), 10 cases (7\%), and 66 cases (48\%), respectively. In the defecography test, the distance of the average fixed perineal descent was $6.2 \pm 2.4 \mathrm{~cm}$, and the average distance of the dynamic perineal descent was $1.1 \pm 1.4 \mathrm{~cm}$.

In regard to fixed perineal descent, the older was the age $(r=$

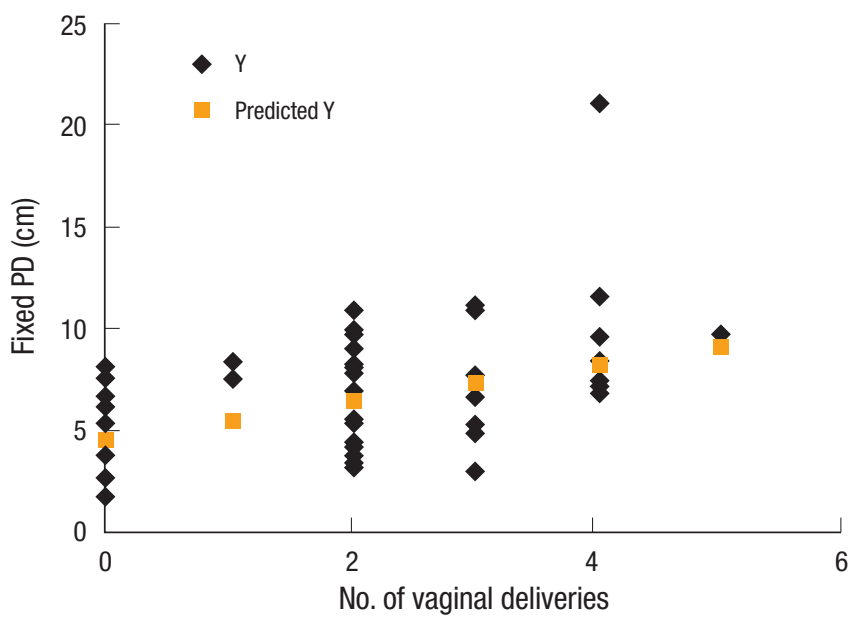

Fig. 2. Vaginal delivery and fixed perineal descent $(\mathrm{PD} ; \mathrm{r}=0.46, \mathrm{P}<$ $0.001)$. 
0.33, $\mathrm{P}<0.001$ ) (Fig. 1), the larger was the diameter of the rectocele $(\mathrm{r}=0.31, \mathrm{P}<0.01)$, and the more frequent was the number of vaginal deliveries $(r=0.46, \mathrm{P}<0.001)$ (Fig. 2$)$, the longer was its length. The length of fixed perineal descent was longer in patients showing symptoms of fecal incontinence than in patients showing symptoms of constipation $(\mathrm{P}<0.05)$ (Table $1)$. In addition, the anorectal angle at rest $(\mathrm{r}=0.67, \mathrm{P}<0.001)$ (Fig. 3), the anorectal angle at squeeze $(\mathrm{r}=0.62, \mathrm{P}<0.001)$, the length of perineal descent at squeeze $(\mathrm{r}=0.41, \mathrm{P}<0.001)$, the length of the puborectalis at rest $(\mathrm{r}=0.49, \mathrm{P}<0.001)$, the length of the puborectalis muscle at squeeze $(\mathrm{r}=0.34, \mathrm{P}<0.001)$, and the value of anal electromyography at squeeze were significantly associated with the length of fixed perineal descent.

In female patients $(\mathrm{P}<0.005)$ (Table 2$)$, rectal intussusception patients $(\mathrm{P}<0.05)$ (Table 3$)$, and rectocele patients $(\mathrm{P}<0.0005)$ (Table 4), the length of dynamic perineal descent was significantly longer. On the other hand, in non-relaxing puborectalis syndrome cases, the length of dynamic perineal descent was significantly shorter $(\mathrm{P}<0.0005)$ (Table 5$)$. The difference in the length of the puborectalis muscle between the push phase and

Table 1. Symptoms and perineal descent

\begin{tabular}{lccc}
\hline Perineal descent $(\mathrm{cm})$ & Constipation & Incontinence & P-value \\
\hline Fixed & $6.1 \pm 2.5$ & $8.3 \pm 2.3$ & 0.0012 \\
Dynamic & $1.1 \pm 1.5$ & $0.3 \pm 1.2$ & 0.117 \\
\hline
\end{tabular}

Table 2. Gender with perineal descent

\begin{tabular}{lccc}
\hline Perineal descent $(\mathrm{cm})$ & Male (27) & Female (109) & P-value \\
\hline Fixed & $6.0 \pm 2.1$ & $6.2 \pm 2.2$ & 0.7599 \\
Dynamic & $0.4 \pm 1.4$ & $1.3 \pm 1.4$ & 0.0016 \\
\hline
\end{tabular}

Table 3. Rectal intussusception and perineal descent (PD)

\begin{tabular}{lccc}
\hline Rectal intussusception & Yes & No & P-value \\
\hline Fixed PD (cm) & $6.2 \pm 2.2$ & $6.1 \pm 2.4$ & 0.892 \\
Dynamic PD (cm) & $1.6 \pm 1.6$ & $1.0 \pm 1.3$ & 0.0482 \\
\hline
\end{tabular}

Table 4. Rectocele and perineal descent (PD)

\begin{tabular}{lccl}
\hline Rectocele & Yes & No & P-value \\
\hline Fixed PD $(\mathrm{cm})$ & $6.3 \pm 2.7$ & $6.0 \pm 2.0$ & 0.42 \\
Dynamic PD (cm) & $1.5 \pm 1.3$ & $0.5 \pm 1.3$ & 0.0001 \\
\hline
\end{tabular}

Table 5. Non-relaxing puborectalis syndrome and perineal descent (PD)

\begin{tabular}{lccc}
\hline $\begin{array}{l}\text { Non-relaxing } \\
\text { puborectalis syndrome }\end{array}$ & Yes & No & P-value \\
\hline Fixed PD (cm) & $6.0 \pm 2.0$ & $6.3 \pm 2.7$ & 0.367 \\
Dynamic PD (cm) & $0.5 \pm 1.2$ & $1.7 \pm 1.4$ & 0.00001 \\
\hline
\end{tabular}

the rest phase $(\mathrm{r}=0.69, \mathrm{P}<0.001)$ and the contrast retention during the push period of the rectocele $(\mathrm{P}<0.0005)$ (Table 6) correlated significantly with dynamic descent.

The anorectal angle during the push phase $(\mathrm{r}=0.61, \mathrm{P}<0.001$, fixed; $\mathrm{r}=0.33, \mathrm{P}<0.001$, dynamic) (Fig. 4) and the length of

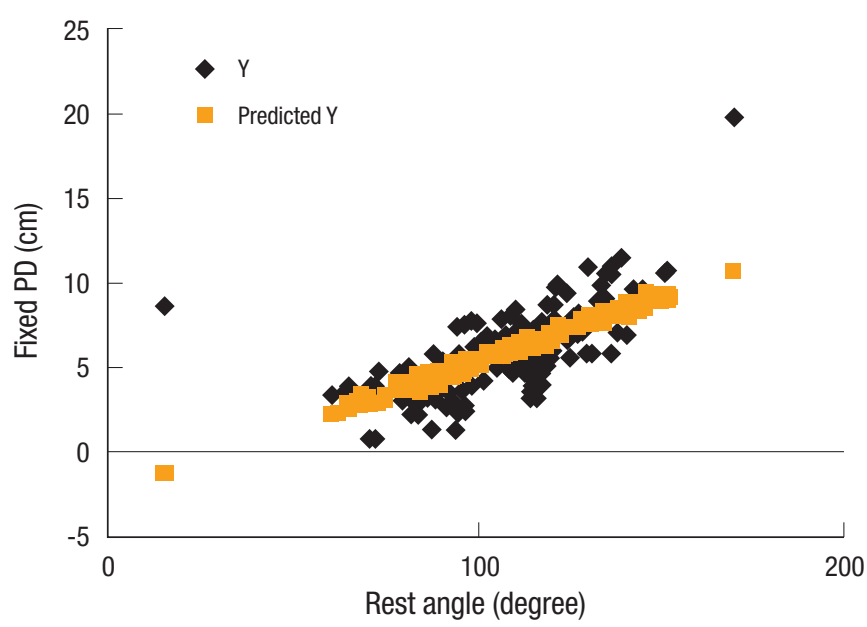

Fig. 3. Rest angle and fixed perineal descent (PD; $r=0.67, \mathrm{P}<0.001)$.

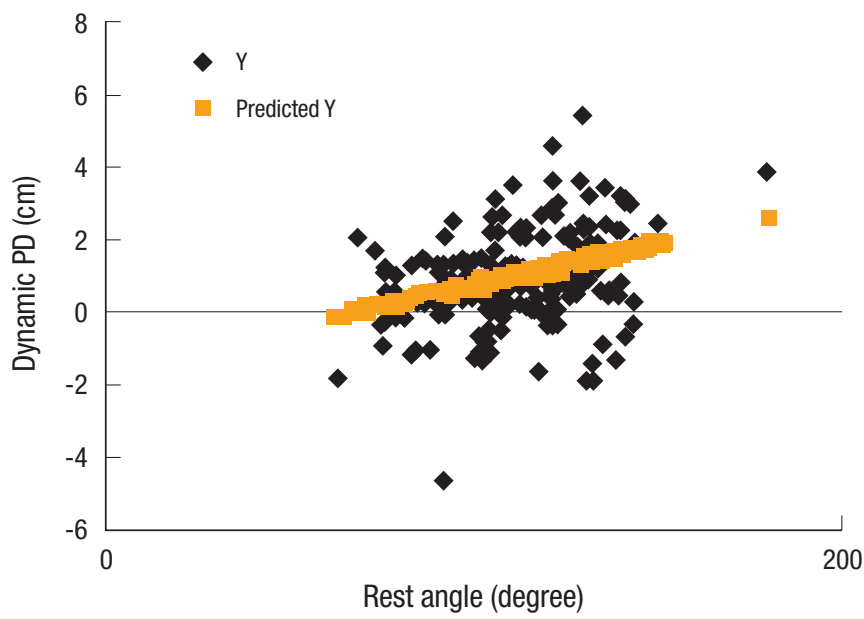

Fig. 4. Push angle and dynamic perineal descent (PD; $\mathrm{r}=0.33, \mathrm{P}<$ $0.001)$.

Table 6. Significant rectocele and perineal descent (PD)

\begin{tabular}{lccl}
\hline Significant rectocele & Yes & No & P-value \\
\hline Fixed PD & $6.5 \pm 2.2$ & $5.9 \pm 2.1$ & 0.172 \\
Dynamic PD & $1.6 \pm 1.2$ & $0.8 \pm 1.5$ & 0.0004 \\
\hline
\end{tabular}

Table 7. Biofeedback and perineal descent (PD)

\begin{tabular}{lccc}
\hline Biofeedback & Success & Failure & P-value \\
\hline Fixed PD (cm) & $6.3 \pm 2.5$ & $5.8 \pm 1.9$ & 0.46 \\
Dynamic PD (cm) & $1.1 \pm 1.5$ & $1.1 \pm 1.1$ & 0.9 \\
\hline
\end{tabular}


the puborectalis muscle during the push phase $(\mathrm{r}=0.48, \mathrm{P}<$ 0.001 , fixed; $r=0.45, P<0.001$, dynamic) correlated significantly with both fixed and dynamic perineal descent. The symptomatic period, the number of defecations, the disease history of pelvic surgery, and dyschezia did not correlate with perineal descent.

One hundred six patients (78\%) responded that the symptoms were improved after biofeedback treatments, and 26 out of those 106 patients (25\%) reported the loss of symptoms. Both fixed perineal descent and dynamic perineal descent of the success group showed no statistically significant differences from the failure group. Thus, neither fixed nor dynamic perineal descent was found to have any effect on the result of biofeedback treatments for pelvic outlet obstruction (Table 7).

\section{DISCUSSION}

Perineal descent is a phenomenon associated with constipation and is considered to be the last stage of excessive straining for defecation continued for several years. Such abnormally increased perineal descent may be considered as the relaxation of the pelvic floor observed in chronic constipation patients. Therefore, other investigators [3-5] have reported that increased perineal descent is a general symptom observed not only in patients with constipation but also in patients with fecal incontinence, idiopathic anal pain syndrome, and solitary rectal ulcer syndrome. It is generally accepted as an secondary phenomenon associated with various diseases causing pelvic outlet obstruction rather than an independent disease inducing chronic constipation. In addition, it is detected not only in constipation patients but also in approximately $75 \%$ of fecal incontinence patients [7]. Excessive straining for defecation for a long time or delivery causes a protrusion of the anterior rectal wall into the anal canal, resulting in the sensation of incomplete defecation and a weakening of the pelvic floor muscles. Such sensation of incomplete defecation and weakening of the pelvic floor muscles result in more powerful and more excessive straining and more weakening of the pelvic floor muscles, eventually leading to a vicious circle [8].

In dyschezia patients, perineal descent and pudendal neuropathy are frequently observed. Parks et al. [9] reported that chronic excessive straining caused entrapment and stretch injury to the nerves due to the gradual descent of the pelvic floor. Other studies reported that the excess descent of the perineal area could stretch the length of pudendal nerves by more than $20 \%$, which was sufficient to induce nervous hindrance $[10,11]$. Finally, injury to the pudental nerves may be a major cause of the weakening of the anal sphincter. The entrapment and stretch theory has been widely supported over the past 15 years. Nonetheless, despite of the existence of such a widely accepted theory, some authors have reported that a significant association could not be elucidated readily. Furthermore, the primary correlation of perineal descent with pudendal neuropathy has been characterized in only two studies. Jones et al. [12] examined 60 idiopathic fecal incontinence patients. The results showed that the level of perineal descent was associated with pudendal neuropathy only when perineal descent was measured at push. Ho and Goh [13] analyzed 141 patients showing perineal descent. Among those patients, constipation was present in 84 patients, neurogenic fecal incontinence in 31 patients, rectal mucosal prolapse in 17 patients, and female stress urine incontinence in 9 patients. The results show that an independent variable that could predict perineal descent at rest was age. Only at push, was the primary correlation of perineal descent to pudendal neuropathy observed. However, Jorge et al. [14] conducted a prospective study on the latent association of increased perineal descent with pudendal neuropathy in 213 dysfunction patients who exhibited constipation (115 patients), idiopathic fecal incontinence (58 patients), chronic incurable anal pain (40 patients), etc. As a result, no patients showed a significant correlation between the extent of descending perineal syndrome and pudendal nerve terminal motor latency (PNTML). Consequently, these authors were unable to demonstrate any correlation between the increased descending perineal syndrome and the functional insufficiency of the pudendal nerve, both of which were assumed to be independent findings. The discrepancy between the two results is thought to be due to the differences in the methods used to measure perineal descent.

Perineal descent may be detected by physical examination, and it can be quantified with the use of physical measurements or defecography. A perineometer with a central latex cylinder that can vertically move freely and measure the movement distance in centimeters was designed by Henry et al. [3]. The cylinder is immobilized by two parallel vertical stainless-steel rods. Patients are positioned in the left incumbent position, the two stainless-steel rods are placed in the ischial tuberosity, and the cylinder is placed in the anal verge. The location of the perineal area at rest is measured by using the scale of the latex cylinder. The patients are asked to perform the action of defecation, and the new location of the perineal area is measured. The difference in the values between the first and the second measurements is the extent of perineal descent. Henry et al. [3] defined cases in which, during the act of defecation, the perineal surface represented by the anal verge descends below the ischial tuberosity as abnormal perineal descent. The device is a clinical way to quantify the extent of perineal descent without exposure to radiation.

Fixed perineal descent and dynamic perineal descent could be imaged by scout photography, which takes images at the time of rest and the action of defecation while performing defecography. In other words, lines were drawn tangent to the posterior verge of the distal rectum and to the central axis of the anal canal, and the angle formed by the two lines was measured as the anorectal angle. Perineal descent is defined as a distance 
reaching the pubococcygeal line at a right angle from the point where these two lines meet. The perineometer could be considered to be an insensitive tool in comparison with defecography. In other words, perineal descent is measured based on the anal verge instead of the anorectal angle, and differences are generated due to the shortening of the anal canal during defecation action. In addition, errors in the distance from the ischial tuberosity to the skin may be made in obese patients, and placing the cylinder precisely without inference of adjacent tissues is difficult. Compared with the perineometer, defecography has advantages in that an actual exclusion of contrast occurs; thus, the measurement during the act of defecation may be more accurate.

Increases in fixed perineal descent show significant correlations to age and the frequency of vaginal deliveries. The result suggests that fixed perineal descent may be a consequence associated with aging or physiological phenomena rather than an independent disease. Nonetheless, more studies are required. On the other hand, the anorectal physiological function test showed increases in fixed perineal descent to be significantly associated with the anorectal angle at rest, the anorectal angle at squeeze, the length of perineal descent at squeeze, the length of the puborectalis muscle, and an increase in the value of anal electromyography, which is thought to reflect the change in each parameter during defecography due to changes in the morphology and the location of the rectum and each pelvic organ with increasing fixed perineal descent.

On the other hand, the increase in dynamic perineal descent correlated with rectal intussusception and the presence of a rectocele and inversely correlated with non-relaxing puborectalis syndrome. These suggest the mutual association of a change in dynamic perineal descent with the development of rectal intussusception and the presence of a rectocele.

Regardless of the methods used to measure perineal descent, perineal descending syndrome is not a disease that can be corrected surgically. For its treatments, the correction of diet habit, laxatives, enemas, biofeedback treatments, the use of support for the perineal area and other non-surgical conservative treatments are performed. Guillemot et al. [15] treated 16 fecal incontinence patients, including 7 perineal descending syndrome patients, with biofeedback treatments and 8 fecal incontinence patients, including 3 perineal descending syndrome patients, with only medications, and the mid- to long-term outcomes from 6 months to 30 months were compared. In patients treated with biofeedback treatments, 12 patients (75\%) showed a lessening of the symptoms. In the patient group treated with only medications, only 1 patient (13\%) showed a lessening of the symptoms. Harewood et al. [16] reported that 39 perineal descending syndrome patients were treated with biofeedback treatments and that excellent results were obtained from $64 \%$ of the patients. In particular, the lesser was the descent level, the better was the treatment outcome. In our cases [17-19] similarly, pelvic outlet obstruction patients associated with perineal descent were treated with biofeedback treatments, and $60-80 \%$ of the patients showed improvement lessening of symptoms. On the other hand, in our cases, the perineal descent level and the treatment score did not show a significant correlation; thus, we conclude that even if perineal descent is conspicuous, biofeedback treatments may be attempted. D'Amico and Angriman [20] reported that a complete cure for perineal descent syndrome is difficult and that treatment should be focused on relieving of symptoms individually according to the cause of the disease of each patient.

In conclusion, fixed perineal descent showed a significant correlation to age, the frequency of vaginal delivery, and the diameter of the rectocele. Dynamic perineal descent showed a significant correlation to rectal intussusception, the rectocele and being female. The level of perineal descent did not affect the outcome of biofeedback treatment for pelvic outlet obstruction; thus regardless of the level of perineal descent, biofeedback may be an effective treatment method.

\section{CONFLICT OF INTEREST}

No potential conflict of interest relevant to this article was reported.

\section{REFERENCES}

1. Porter NH. A physiological study of the pelvic floor in rectal prolapse. Ann R Coll Surg Engl 1962;31:379-404.

2. Parks AG, Porter NH, Hardcastle J. The syndrome of the descending perineum. Proc R Soc Med 1966;59:477-82.

3. Henry MM, Parks AG, Swash M. The pelvic floor musculature in the descending perineum syndrome. Br J Surg 1982;69:470-2.

4. Bartolo DC, Read NW, Jarratt JA, Read MG, Donnelly TC, Johnson AG. Differences in anal sphincter function and clinical presentation in patients with pelvic floor descent. Gastroenterology 1983;85:68-75.

5. Snooks SJ, Nicholls RJ, Henry MM, Swash M. Electrophysiological and manometric assessment of the pelvic floor in the solitary rectal ulcer syndrome. Br J Surg 1985;72:131-3.

6. Kuijpers HC, Bleijenberg G. The spastic pelvic floor syndrome. A cause of constipation. Dis Colon Rectum 1985;28:669-72.

7. Read NW, Bannister JJ. Anorectal manometry: techniques in health and anorectal disease. In: Henry MM, Swash M, editors. Coloproctology and the pelvic floor: pathophysiology and management. London: Butterworths; 1985. p. 65-87.

8. Hardcastle JD. The descending perineum syndrome. Practitioner 1969;203:612-9.

9. Parks AG, Swash M, Urich H. Sphincter denervation in anorectal incontinence and rectal prolapse. Gut 1977;18:656-65.

10. Sunderland S. Nerves and nerve injuries. New York: Churchill Livingstone; 1978. 
11. Henry MM, Parks AG, Swash M. The anal reflex in idiopathic faecal incontinence: an electrophysiological study. Br J Surg 1980; 67:781-3.

12. Jones PN, Lubowski DZ, Swash M, Henry MM. Relation between perineal descent and pudendal nerve damage in idiopathic faecal incontinence. Int J Colorectal Dis 1987;2:93-5.

13. Ho YH, Goh HS. The neurophysiological significance of perineal descent. Int J Colorectal Dis 1995;10:107-11.

14. Jorge JM, Wexner SD, Ehrenpreis ED, Nogueras JJ, Jagelman DG. Does perineal descent correlate with pudendal neuropathy? Dis Colon Rectum 1993;36:475-83.

15. Guillemot F, Bouche B, Gower-Rousseau C, Chartier M, Wolschies E, Lamblin MD, et al. Biofeedback for the treatment of fecal incontinence. Long-term clinical results. Dis Colon Rectum 1995; 38:393-7.
16. Harewood GC, Coulie B, Camilleri M, Rath-Harvey D, Pemberton JH. Descending perineum syndrome: audit of clinical and laboratory features and outcome of pelvic floor retraining. Am J Gastroenterol 1999;94:126-30.

17. Baek SM, Kim NH, Hwang YH, Kim KR, Choi KP. Outcome and prognostic factors associated with poor outcome of biofeedback therapy for constipated patients with non-relaxing puborectalis syndrome. J Korean Soc Coloproctol 2003;19:74-81.

18. Kim BS, Hwang YH, Choi KP. Biofeedback therapy for patients with rectocele. J Korean Soc Coloproctol 2001;17:69-75.

19. Kwon SH, Hwang YH, Choi KP. Outcome and predictors of biofeedback therapy for patients with internal rectal intussusception. J Korean Soc Coloproctol 2002;18:379-85.

20. D’Amico DF, Angriman I. Descending perineum syndrome: iatrogenic or spontaneous pathology? Chir Ital 2000;52:625-30. 